\title{
Perceived cost potential field cellular automata model with an aggregated force field for pedestrian dynamics
}

\author{
Xiao-Xia Jian, ${ }^{\mathrm{a}, \mathrm{b}, \mathrm{d}}$, S.C. Wong ${ }^{\mathrm{c}, *}$, Peng Zhang ${ }^{\mathrm{b}, \mathrm{d}}$, Keechoo Choi ${ }^{\mathrm{e}}$, Hong Li ${ }^{\mathrm{f}}$, \\ Xiaoning Zhang ${ }^{\mathrm{a}}$ \\ ${ }^{a}$ School of Economics and Management, Tongji University, \\ Shanghai 200092, P.R. China \\ ${ }^{b}$ Shanghai Institute of Applied Mathematics and Mechanics, Shanghai University, \\ Shanghai, 200072, P.R. China \\ ${ }^{c}$ Department of Civil Engineering, The University of Hong Kong, Pokfulam Road, \\ Hong Kong SAR, P.R. China \\ ${ }^{d}$ Shanghai Key Laboratory of Mechanics in Energy Engineering \\ ${ }^{e}$ Department of Transportation Engineering, TOD-based Sustainable Urban \\ Transportation Center, Ajou University, Korea \\ ${ }^{f}$ School of Mathematical Science, Inner Mongolia University, \\ Hohhot, 010021, P.R. China
}

\begin{abstract}
This paper proposes a perceived potential field and an aggregated force field for the navigation of pedestrians in a walking domain with poor visibility or complex geometries. While the former field used in uncrowded cells simply reflects the pedestrians' desire to minimize their travel costs, the latter field used in crowded cells suggests much stronger interaction between pedestrians. Compared with a formulation that does not include the latter field, the proposed model displays an advantage in simulating over-crowded pedestrian flows, e.g. at the front of a bottleneck or at a left/right turn in a corridor; the simulated phenomena, including phase transitions and fundamental diagrams, agree well with the observations and studies in the literature.
\end{abstract}

Keywords:

Visibility, Complex geometries, Bottlenecks, Over-crowded situation, Fundamental diagrams, Back-moving jams

\footnotetext{
${ }^{*}$ Corresponding author. Tel: +852-2859-1964; Fax: +852-2559-5337

Email address:hhecwsc@hku.hk (S.C. Wong)
} 


\section{Introduction}

Pedestrian dynamics are important in the analysis and design of transportation facilities, walkways, and public buildings. Recently, various models have been developed to simulate walking behavior and investigate macroscopic and microscopic characteristics of pedestrian dynamics in complex scenarios; many of the typical collection effects (Burstedde et al., 2001; Kirchner and Schadschneider, 2002) and self-organized phenomena (Helbing et al., 2005) described have reflectedthe microscopic interactions of individuals and the effects of environmental information, which paves the way for further development of pedestrian dynamics.

Typical pedestrian flow models include the macroscopic continuum model (Hughes, 2002; Hoogendoorn and Bovy, 2004; Huang et al., 2009; Xia et al., 2009; Jiang et al., 2010; Xiong et al., 2011), the microscopic social force model (Helbing and Molnár, 1995; Moussaïd et al., 2011), and the cellular automata (CA) model (Burstedde et al., 2001; Kirchner and Schadschneider, 2002; Huang and Guo, 2008; Kuang et al., 2008; Kretz, 2010; Zhang etal., 2012). The continuum model describes pedestrian flow using partial differential equations, in which case the pressure inside a crowd reflects the compressibility of the crowd (Lee and Hughes, 2005) regardless of whether there is physical contact between the pedestrians. Physical contact can usually be avoided if the density is lower than $5.6 \mathrm{ped} / \mathrm{m}^{2}$; however, contact becomes inevitable as the density increases and the accumulated pushing force takes effect. The social force model treats pedestrians as particles and establishes the acceleration of these particles using Newton's second law, the forces reflecting self-driven power toward destinations and interactions between pedestrians. The CA model divides the walking domain into cells, and each pedestrian remains in place or moves to an empty neighboring cell with a certain probability at each time step.

The CA model has attracted much attention in simulating complex pedestrian collection effects, due to its simple update rules. In particular, the floor field CA model (Burstedde et al., 2001; Kirchner and Schadschneider, 2002; Varas et al., 2007) introduces static and dynamic floor fields for navigating pedestrian motion. The value of the static floor field in a cell is measured by the distance between the cell and the destination, which is unchanging across the simulation. The value of the dynamic floor field in a cell is measured by a virtual trace left by pedestrians, which may decay and diffuse at each time step, and reflects the interactions between pedestrians. See also Huang and Guo (2008), Kretz et al. (2010), and Guo and Huang (2012). Another typical CA model (Zhang et al., 2012) incorporates the static and dynamic factors in a cost distribution, and the cost potentials in all cells are derived by solving an eikonal equation in each time step. Accordingly, a pedestrian remains in place or moves to an empty neighboring cell so 
thatthe reduction in potential is maximized. Such a path-choice strategy actually helps the pedestrian to minimize his/her cost to the destination, and thus the randomness in determining the probabilities for occupation is greatly reduced. This is reasonable under the assumption that all pedestrians are familiar with the surroundings.

To model a pedestrian crowd with a higher density or compressibility, the CA model is extended such that a cell is allowed to be occupied by, at most, two pedestrians (Xie et al., 2012). Moreover, a microscopic force field (Henein and White, 2007, 2010) is introduced to describe interpersonal contacts. To model pedestrian flow in a domain with complex geometries, the degree of visibility, which influences pedestrians' path-choice strategy, is considered (Moussaïd et al., 2011; Zeng et al., 2011; Guo et al., 2012; Xu and Huang, 2012). The floor field CA model is used to simulate pedestrian flow with multiple exits (Huang and Guo, 2008, Xu and Huang, 2012), through a corridor with bottlenecks (Kretz, 2010) or with corners (Kretz, 2009; Bönisch and Kretz, 2009; Steffen and Seyfried, 2009; Zeng et al., 2011; Guo and Tang, 2012), in a T-junction walking domain (Peng and Chou, 2011), and in a room with obstacles (Alizadeh, 2011).

In this paper, the cost potential field (Zhang et al., 2012) is extended to simulate pedestrian flow in a walking facility with complex geometries, in which visibility is considered. For the extension, a perceived cost potential field is constructed by taking its value in a cell to be the weighted average between that of the cost potential in Zhang et al. (2012) and that of a memory potential. The memory potential is measured by the distance or displacement from the cell to the destination, which is actually a degenerated cost potential, such that the surroundings are completely invisible and pedestrians are only able to conceive of the path to their destination according to their experience. Thus, the weight on the cost potential is taken to be a measure of visibility.

We further introduce pushing forces for the simulation of crowded or over-crowded pedestrian flow, which is increasing of the density and decreasing of the visibility in the surroundings, pointing to the destination from referred cells. Moreover, we assume that these forces aggregate in a certain area behind a pedestrian with respect to that pedestrian, forming an aggregated force field that can take effect due to contact or psychological factors that push the pedestrian into a nonempty neighboring cell when a threshold value is exceeded. As a cell is allowed to be occupied by, at most, 2 pedestrians, the maximal density herein extends to $12.5 \mathrm{ped} / \mathrm{m}^{2}$, which is twice that in most CA models (Burstedde et al., 2001; Kirchner and Schadschneider, 2002; Zhang et al., 2012).

The general path-choice strategy of the proposed model is as follows. For an occupied cell (one or two pedestrians) that is uncrowded, a pedestrian in the cell navigates using the perceived cost field; that is, he/she remains in place or moves to an empty neighboring cell, reducing the perceived cost potential to the fullest extent. This rule is the same as that in Zhang et al. (2012), except that the cost potential is replaced by the perceived cost 
potential and the cell might be occupied by two pedestrians. The formulation is reduced to that in Zhang et al. (2012) if the walking domain (including the destination) is completely visible and a cell can only be occupied by, at most, one pedestrian. For an occupied cell that is crowded, which means that all of its neighboring cells are also occupied and the local density is greater than $6.25 \mathrm{ped} / \mathrm{m}^{2}$, the aggregated force field is used for navigation.

The remainder of this paper is organized as follows. In Section 2, we formulate the perceived cost potential field CA model and the aggregated force field. In Section 3, two scenarios are presented to show the robustness of the model in simulating crowded or even over-crowded pedestrian flow in a corridor, with a bottleneck, and with a $90^{\circ}$ corner, respectively. The conclusions are presented in Section 4.

\section{Model description}

The walking domain is divided into cells of size $0.4 \mathrm{~m} \times 0.4 \mathrm{~m}$. We assume that a cell can be occupied by, at most, two pedestrians, which suggests a maximal density of $12.5 \mathrm{ped} / \mathrm{m}^{2}$. This is very close to the $11 \mathrm{ped} / \mathrm{m}^{2}$ suggested by the field study (Helbing et al., 2007; Moussaïd et al., 2011), and twice the $6.25 \mathrm{ped} / \mathrm{m}^{2}$ derived by assuming that a cell can be occupied by, at most, one pedestrian.

(a)

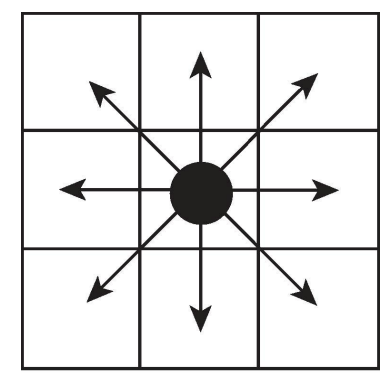

(b)

\begin{tabular}{|l|l|l|}
\hline$p_{-1,-1}$ & $p_{-1,0}$ & $p_{-1,1}$ \\
\hline$p_{0,-1}$ & $p_{0,0}$ & $p_{0,1}$ \\
\hline$p_{1,-1}$ & $p_{1,0}$ & $p_{1,1}$ \\
\hline
\end{tabular}

Fig. 1. (a) An occupied cell $(0,0)$ and its eight neighboring cells; (b) the corresponding probabilities for occupation in the next update step. Note that the cell can be occupied by, at most, two pedestrians.

The dimensionless density is defined as $\rho(x, y, t)=\sum_{(x, y)} \in \mathrm{D} x, y n(x, y, t) /\left(2 \cdot\left|D_{x, y}\right|\right)$, where $D_{x, y}$ represents the local domain around $(x, y)$, including 25 cells, and $\left|D_{x, y}\right|$ denotes the number of cells in $D_{x, y}$. Considering that the mean velocity of a pedestrian is approximately $1 \mathrm{~m} / \mathrm{s}$, we choose the maximal velocity to be $v_{\max }=1 \mathrm{~m} / \mathrm{s}$. This choice has the advantages of simplicity and high simulation speed. A detailed discussion of pedestrian velocity can be found in Burstedde et al. (2001), Schadschneider (2002), and Kirchner et al. (2004). 
As mentioned above, a pedestrian at $(x, y)=(0,0)$ might remain in place or move to a neighboring cell. The direction of motion and the probabilities $p_{i, j}$ for occupation are shown in Fig. 1. According to the probabilities and the rules for resolving conflicts, the positions of all pedestrians are updated in parallel from time steps $t$ to $t+1$. Here, $p_{i, j}$ values are determined by the perceived cost potential field or the aggregated pushing force field, which together with the resolution of conflicts are discussed in the following sections.

\subsection{Perceived cost potential}

We assume that a pedestrian at $(x, y)$ desires to arrive at the destination or exit $\Gamma_{0}$ with a minimized traveling cost. Such a desired cost $\phi(x, y, t)$ at time $t$ is called the cost potential (Hughes, 2002; Hoogendoorn and Bovy, 2004; Huang et al., 2009; Jiang et al., 2010; Xiong et al., 2011; Zhang et al., 2012). Let the cost distribution at time $t$ be denoted by $c(x, y, t)$, such that the cost equals $c(x, y, t) \mathrm{d} s$ for pedestrians at $(x, y)$ traveling a sufficiently small distance $\mathrm{d} s$. Assume that $c(x, y, t)$ is related to the density $\rho(x, y, t)$ through

$$
c(x, y, t)=\frac{1}{v_{e}(\rho(x, y, t))}+g(\rho(x, y, t)),
$$

where $v_{\mathrm{e}}(\rho)$ is the traveling speed, through which the cost is associated with the traveling time of pedestrians and $g(\rho(x, y, t))$ is the increase in $\rho$ with $g(0)=0$, through which the cost is associated with pedestrians' discomfort. Then, the potential cost $\phi(x, y, t)$ satisfies the following eikonal equation:

$$
\begin{array}{ll}
\|\nabla \phi(x, y, t)\|=c(x, y, t), & (x, y) \in \Omega, \\
\phi\left(x_{0}, y_{0}, t\right)=0, & \left(x_{0}, y_{0}\right) \in \Gamma_{0} .
\end{array}
$$

This can be verified by showing that the solution to Eq. (2) is truly the desired minimal cost for traveling from $(x, y)$ to $\Gamma_{0}$ at time $t$ (see Zhao (2005) and references therein for the numerical solution and discussion on the existence and uniqueness of the solution to Eq. (2)). For a path $l$ from $(x, y)$ to $\left(x_{0}, y_{0}\right) \in \Gamma_{0}$, the expected traveling cost is

$$
\begin{aligned}
& \int_{l} c(x, y, t) \mathrm{d} s \geq \int_{l} c(x, y, t)\left(-\frac{\nabla \phi}{|\nabla \phi|}\right) \cdot \mathbf{d} \mathbf{s}=-\int_{l} \nabla \phi \cdot \mathbf{d s} \\
& =-\left(\phi\left(x_{0}, y_{0}, t\right)-\phi(x, y, t)\right)=\phi(x, y, t),
\end{aligned}
$$

where the vector $\mathbf{d s}$ is tangential to the path $l$, and the first equality holds if and only if $\mathbf{d s} \|-\nabla \phi$. This indicates that $\phi(x, y, t)$ is indeed the desired minimum traveling cost, which corresponds to the path parallel to the negative gradient $-\nabla \phi(x, y, t)$. To apply the aforementioned path-choice strategy in the forthcoming discussion, we replace $v_{\mathrm{e}}(\rho)$ by $v_{\max }=1$, as speed is constant in the CA model. The resulting cost potential is denoted by 
$\phi_{a}(x, y, t)$, which is actually the same as that in Zhang et al. (2012).

To reflect the influence of visibility on the path-choice strategy, we introduce a memorized cost potential $\phi_{m}(x, y, t)$, which is derived by setting the discomfort term of Eq. (1) to be zero; that is, we obtain $\phi_{m}(x, y, t) \equiv \phi_{a}(x, y, t)$ by setting $g(\rho) \equiv 0$. Here, $\phi_{m}(x, y, t)$ is actually a measure of the distance or, more precisely, the minimized spatial steps from $(x, y)$ to $\Gamma_{0}$. The perceived cost potential $\phi_{p}(x, y, t)$ is taken to be a convex combination of $\phi_{a}(x, y, t)$ and $\phi_{m}(x, y, t)$,

$$
\phi_{p}(x, y, t)=\omega(x, y) \cdot \phi_{a}(x, y, t)+(1-\omega(x, y)) \cdot \phi_{m}(x, y, t)
$$

where the weight $\omega(x, y)$ measures the visibility. It is obvious that $\phi_{p}(x, y, t)$ is identical to $\phi_{a}(x, y, t)$ when the visibility is perfectly good, with $\omega(x, y)=1$. Conversely, $\phi_{p}(x, y, t)$ is identical to $\phi_{m}(x, y, t)$ if the visibility is extremely poor, with $\omega(x, y)=0$. In this case, we assume that pedestrians are familiar with the walking domain so that they are able to find the shortest path to their destination, according to their memory or experience; however, we assume that they are not able to experience any discomfort (reflected by $g(\rho)$ in Eq. (1)) because they cannot see other pedestrians in the surroundings.

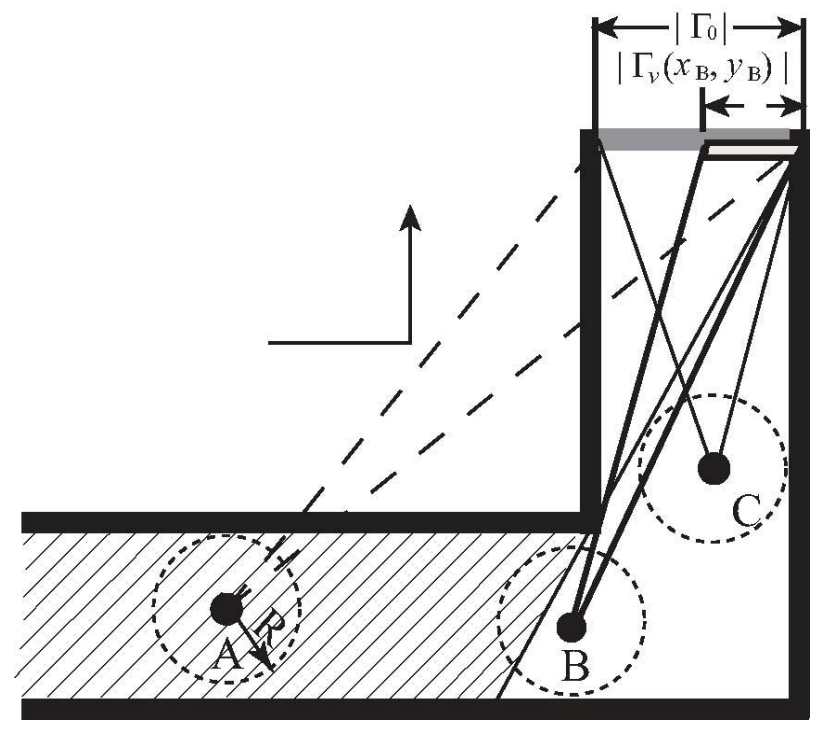

Fig. 2. The related parameters of the visibility definition in Eq. (5). The dotted circle with the radius $R$ surrounding each pedestrian is the visible range. When $R$ is large enough, the visibility of the exit for each pedestrian is shown by the lines of sight. For example, for pedestrian $\mathbf{A}$ in the shaded portion where $\left|\Gamma_{v}\right|=0$, all the lines of sight (between the two dashed lines starting from $\mathbf{A}$ ) are blocked by obstacles (black squares); for pedestrian $\mathbf{B}$, in the portion where $0<\left|\Gamma_{v}\right| \leq\left|\Gamma_{0}\right|$, the line of sight (between two solid black lines starting from 
B) is partially obstructed by obstacles and pedestrian $\mathbf{B}$ can see a part $\Gamma_{v}\left(x_{\mathrm{B}}, y_{\mathrm{B}}\right)$ (bold, light gray line surrounded by black frame) of the exit $\Gamma_{0}$ (bold gray line); pedestrian $\mathbf{C}$, whose line of sight (between the two solid grey lines starting from $\mathbf{C}$ ) is totally unobstructed, can see the whole exit $\Gamma_{0}$. The black arrow indicates the direction of motion.

Let $R(x, y)$ denote the radius of the visible area centered at $(x, y), \Gamma_{v}(x, y)$ the visible part(s) of the exit $\Gamma_{0}$ at $(x, y)$, and $d(x, y)$ the shortest visible distance from $(x, y)$ to $\Gamma_{v}(x, y)$. We then assume that $\omega(x, y)$ increases with increasing $R$ and the length $\left|\Gamma_{v}(x, y)\right|$, and decreases withincreasing $d(x, y)$. One description for these dependencies is expressed by the following formula:

$$
\omega(x, y)=\exp \left(-\frac{\left|\Gamma_{0}\right|-\left|\Gamma_{v}(x, y)\right|}{\left|\Gamma_{0}\right|}\right) \exp \left(-\frac{d(x, y)}{R(x, y)}\right),
$$

where it is obvious that $0<\omega \leq 1$, with $\omega \rightarrow 0$ for $R \rightarrow 0$, and $\omega \rightarrow 1$ for $R \rightarrow \infty$ and $\Gamma_{v}(x, y) \rightarrow \Gamma_{0}(x, y)$. See Fig. 2 for parameter meanings.

\subsection{Aggregated force field}

The magnitude of the pushing force produced at $(x, y, t)$ is defined as

$$
f(x, y, t)=\frac{n(x, y, t)}{\phi_{p}(x, y, t)-\phi_{m}(x, y, t)}=\frac{n(x, y, t)}{\left(\phi_{a}(x, y, t)-\phi_{m}(x, y, t)\right) \omega(x, y)},
$$

where $n(x, y, t) \leq 2$ is the number of pedestrians in the cell $(x, y)$ and at time $t$. The physics of Eq. (6) is interpreted as follows. The more pedestrians in cell $(x, y)$, the larger the pushing force therein; in particular, we have $f=0$ for $n=0$. Moreover, the larger the difference between the perceived cost potential $\phi_{p}(x, y, t)$ and the memorized cost potential $\phi_{m}(x, y, t)$, the less incentive to push. This is because a larger difference between $\phi_{p}$ and $\phi_{m}\left(\phi_{p}>\phi_{m}\right)$ usually suggests a higher density on the way to the destination, in which case the pedestrians are aware of the congestion ahead and feel that pushing hard is useless. More influentially, $f$ decreases with increasing $\omega$, which means that pedestrians with poorer visibility have a stronger incentive to push. As pushing behavior is closely associated with poor visibility, in which case the memorized cost potential $\phi_{m}$ is dominant, we assume that the pushing direction is parallel to $-\nabla \phi_{m}(x, y, t)$. Therefore, the pushing force at $(x, y, t)$ is given by:

$$
\vec{f}(x, y, t)=-\frac{\nabla \phi_{m}(x, y, t)}{\left|\nabla \phi_{m}(x, y, t)\right|} f(x, y, t),
$$

where the unit vector is re-denoted by $\vec{l}_{m}(x, y, t)=\left(l_{m}^{1}(x, y, t), l_{m}^{2}(x, y, t)\right)$.

An aggregated force field is taken into account as follows. At each time step, the 
pushing force is repeatedly transmitted from individual to individual through interpersonal contacts (or nervousness) within a crowded region (Fruin, 1993). Therefore, each pedestrian in the region experiences the pushing forces from other pedestrians behind $\mathrm{him} /$ her instantaneously. Given an occupied cell $(x, y)$ within the region, we assume that the forces behind in a subregion $\mathfrak{C}(x, y, t)$ are aggregated to act on the cell. Therefore, the aggregated force at $(x, y, t)$ is given by:

$$
\vec{F}(x, y, t)=\sum_{(\bar{x}, \bar{y}) \in \mathfrak{C}(x, y, t)} \vec{f}(\bar{x}, \bar{y}, t),
$$

and the two components of $\vec{F}(x, y, t)$ are simply given as

$$
\begin{aligned}
& F^{1}(x, y, t)=\sum_{(\bar{x}, \bar{y}) \in \mathfrak{C}(x, y, t)} f(\bar{x}, \bar{y}, t) l_{m}^{1}(\bar{x}, \bar{y}, t), \\
& F^{2}(x, y, t)=\sum_{(\bar{x}, \bar{y}) \in \mathfrak{C}(x, y, t)} f(\bar{x}, \bar{y}, t) l_{m}^{2}(\bar{x}, \bar{y}, t) .
\end{aligned}
$$

Here, $\mathfrak{C}(x, y, t) \subseteq \mathfrak{C}_{\max }(x, y, t)$ is a collection of cells behind the cell $(x, y)$, which is defined as follows. We first define the set $\mathfrak{C}_{\max }(x, y, t)$ as follows:

(a)

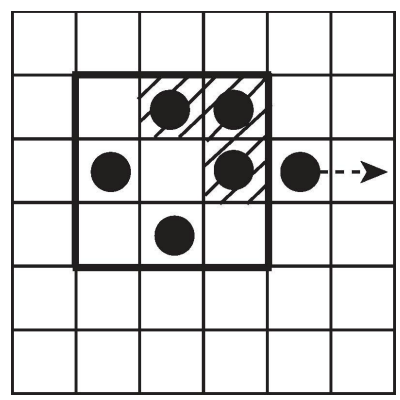

(b)

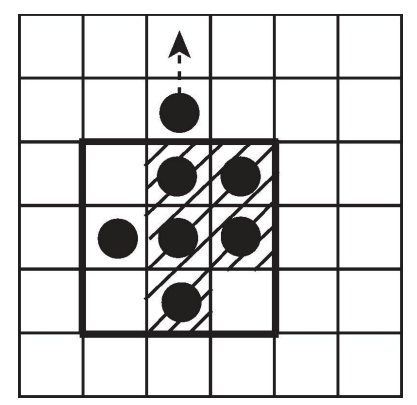

(c)

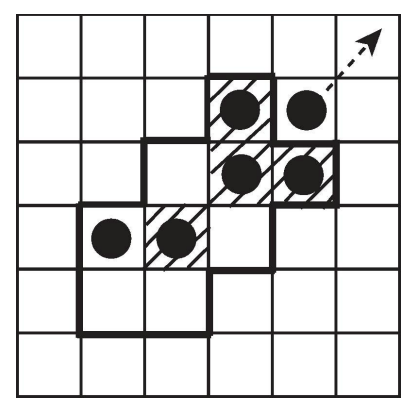

Fig. 3. A rear group corresponding to the desired direction along (a) level direction; (b) vertical direction; and (c) diagonal direction. All positions in each area (surrounded by the thick black lines) make up the set $\mathfrak{C}_{\text {max }}(x, y, t)$, the shadow parts are set $\mathfrak{C}(x, y, t)$, and each arrow with the dotted lines points in the direction of $-\nabla \phi_{m}(x, y, t)$.

(i) $\mathfrak{C}_{\max }(x, y, t)=\left\{(\bar{x}, \bar{y}) \mid(\bar{x}-x) \cdot\left(-l_{m}^{1}\right) \in[0,[R]],(\bar{y}-y) \in[-1,1]\right\}$, if $\vec{l}_{m}(x, y, t)$ is along the level direction (right- or leftward) with $\left|l_{m}^{1}\right|=1$ and $\left|l_{m}^{2}\right|=0$, (Fig. 3(a));

(ii) $\mathfrak{C}_{\max }(x, y, t)=\left\{(\bar{x}, \bar{y}) \mid(\bar{y}-y) \cdot\left(-l_{m}^{2}\right) \in[0,\lfloor R]],(\bar{x}-x) \in[-1,1]\right\}$, if $\vec{l}_{m}(x, y, t)$ is along the vertical direction (up- or downward) with $\left|l_{m}^{1}\right|=0$ and $\left|l_{m}^{2}\right|=1$, (Fig. 3(b)); and 
(iii) $\mathfrak{C}_{\max }(x, y, t)=\left\{(\bar{x}, \bar{y})\left|(\bar{x}-x) \cdot\left(-l_{m}^{1}\right) \in[0,|R / \sqrt{2}|],\right|(\bar{x}-x)-(\bar{y}-y) \mid \leq 1\right\}$, if

$\vec{l}_{m}(x, y, t)$ is along the diagonal direction (left-upward, left-downward, right-upward, or right-downward) with $\left|l_{m}^{1}\right|+\left|l_{m}^{2}\right|=\sqrt{2}$, (Fig. 3(c)).

Here, $\lfloor R\rfloor$ is the largest integer not greater than $R$. We then assume that a cell $(\bar{x}, \bar{y})$ belongs to $\mathfrak{C}(x, y, t)$ if and only if $(\bar{x}, \bar{y})$ and at least one of its neighboring cells along the direction $-\vec{l}_{m}(x, y, t)$ or $\vec{l}_{m}(x, y, t)$ are occupied. The cells belonging to $\mathfrak{C}(x, y, t)$ are shaded in Fig. 3 and Figs. 3(a), 3(b), and 3(c) correspond to the level, vertical, and diagonal directions, respectively.

It is obvious that the pushing force in a cell depends on the density around that cell. After updating pedestrians' positions, changes in density cause changes in pushing force. This process can be used to describe the transmission or propagation of pushing force from one pedestrian (or position) to another, which reflects well the dynamic nature of pedestrian crowds.

\subsection{Update rules}

General rules for determining the occupation probabilities $p_{i, j}$ in Fig. 1 are as follows:

1. If the occupied cell $(0,0)$ is uncrowded, then the perceived cost potential field is used to determine a pedestrian's motion.

2. Otherwise, if the cell is crowded, the aggregated force field is used for the navigation.

Here, an occupied cell is crowded if (i) the eight neighboring cells are occupied, and (ii) the density of the cell exceeds $6.25 \mathrm{ped} / \mathrm{m}^{2}$. Otherwise, the cell is uncrowded. We note that the threshold $6.25 \mathrm{ped} / \mathrm{m}^{2}$ is the maximal density in the case that a cell is allowed to be occupied by at most one pedestrian.

\subsubsection{Probabilities $p_{i, j}$ determined by perceived cost potential field}

For a pedestrian in an uncrowded cell $(0,0)$, the rule for his/her to update position can be simply interpreted as follows. A neighboring cell is his/her target only if this cell is empty and if moving to this cell could reduce his/her potential to the fullest extent. If there is more than one such neighboring cell, then they share the same probability to become a target cell; if there is no such neighboring cell, the pedestrian remains in place. More precisely, the probabilities $p_{i, j}$ are determined by the following procedures:

1. Compute the difference quotient $\delta \phi_{p ; i, j}(0,0) \equiv\left(\phi_{p}(i, j)-\phi_{p}(0,0)\right) / \boldsymbol{l}_{i, j}$ for $(i, j) \in S_{0}^{\mathrm{I}}=$ 
$\{(i, j) \mid(i, j)$ is empty $\}$, where $l_{i, j}(=1$ or $\sqrt{2})$ is the distance between the cells $(i, j)$ and $(0,0)$;

2. Define the set

$$
S_{m}^{\mathrm{I}}=\left\{(i, j) \mid \delta \phi_{p ; i, j}(0,0)=\delta \phi_{p ; \min }(0,0)\right\} \subseteq S_{0}^{\mathrm{I}},
$$

where $\delta \phi_{p ; \min }(0,0)=\min _{(i, j) \in S_{0}^{1}} \delta \phi_{p ; i, j}(0,0)$;

3. Determine the probabilities by

$$
\begin{aligned}
& p_{i, j}=\left\{\begin{array}{cc}
1 /\left|S_{m}^{\mathrm{I}}\right|, & (i, j) \in S_{m}^{\mathrm{I}}, \\
0, & (i, j) \notin S_{m}^{\mathrm{I}},
\end{array} \text { if } S_{m}^{\mathrm{I}} \neq \varnothing, \text { and } \delta \phi_{p ; \text { min }}(0,0)<0,\right. \\
& p_{i, j}= \begin{cases}1, & (i, j)=(0,0), \\
0, & (i, j) \neq(0,0),\end{cases}
\end{aligned}
$$

where $\left|S_{m}^{\mathrm{I}}\right|$ is the number of elements in $S_{m}^{\mathrm{I}}$.

\subsubsection{Probabilities $p_{i, j}$ determined by aggregated force field}

For a pedestrian in a crowded cell $(0,0)$, we note that no neighboring cells are empty. In this case, we assume that a neighboring cell $(i, j)$ is his/her target cell only if (i) this cell is occupied by only one pedestrian, and (ii) the projection of the aggregated force $\vec{F}_{0,0}$ onto the direction of motion toward this cell exceeds a threshold (e.g. $F_{0,0}^{*}=5 f_{0,0}$ ) and attains the maximum among those cells satisfying (i). If there is more than one such cell, then they share the same probability to become a target cell; if there is no such cell, the pedestrian will remain in place. More precisely, the probabilities $p_{i, j}$ are determined by the following procedures:

1. Define the set $S_{0}^{\mathrm{II}}=\{(i, j) \mid(i, j)$ is occupied by one pedestrian $\}$;

2. Define the set

$$
S_{m}^{\mathrm{II}}=\left\{(i, j) \mid F_{i, j}^{P}=\max _{(i, j) \in S_{0}^{\mathrm{II}}} F_{i, j}^{P} \equiv F^{P}(0)\right\} \subseteq S_{0}^{\mathrm{II}},
$$

where $F_{i, j}^{P}=\vec{F}_{0,0} \cdot \vec{z}_{i, j}$ is the projection of $\vec{F}_{0,0}$ onto $\vec{z}_{i, j}$, and $\vec{z}_{i, j}=((i, j)-(0,0)) / l_{i, j}$ is the direction of motion toward $(i, j)$;

3. Determine the probabilities

$$
\begin{aligned}
& p_{i, j}=\left\{\begin{array}{cl}
1 /\left|S_{m}^{\mathrm{II}}\right|, & (i, j) \in S_{m}^{\mathrm{II}}, \\
0, & (i, j) \notin S_{m}^{\mathrm{II}},
\end{array} \text { if } S_{m}^{\mathrm{II}} \neq \varnothing, \text { and } F_{i, j}^{P} \geq F_{0,0}^{*},\right. \\
& p_{i, j}=\left\{\begin{array}{ll}
1, & (i, j)=(0,0), \\
0, & (i, j) \neq(0,0),
\end{array}\right. \text { otherwise, }
\end{aligned}
$$


where $\left|S_{m}^{\mathrm{II}}\right|$ is the number of elements in $S_{m}^{\mathrm{II}}$.

\subsubsection{Resolution of conflicts}

A cell $(i, j)$ that is empty or occupied by one pedestrian can be a target cell of $M$ pedestrians in neighboring cells denoted by $\left(0^{(k)}, 0^{(k)}\right)$. This gives rise to an occupation conflict in the case where $M \geq 2$; theoretically, $M$ could be up to 16 . To resolve a possible conflict, we determine the probabilities for occupation as follows:

$$
p\left(0^{(k)}, 0^{(k)}\right)=\left\{\begin{array}{cc}
1 /\left|S_{m m}^{c}\right|, & \text { if } M \geq 1, \text { and }\left(0^{(k)}, 0^{(k)}\right) \in S_{m m}^{c}, \\
0, & \text { otherwise, }
\end{array}\right.
$$

where $c=\mathrm{I}$ is for $(i, j)$ being an empty cell, and $c=\mathrm{II}$ is for $(i, j)$ being occupied by one pedestrian; giving the set

$$
S_{m m}^{\mathrm{I}}=\left\{\left(0^{k}, 0^{(k)}\right) \mid \delta \phi_{p ; \text { min }}\left(0^{k}, 0^{(k)}\right)=\min _{k} \delta \phi_{p ; \text { min }}\left(0^{k}, 0^{(k)}\right)\right\},
$$

and the set

$$
S_{m m}^{\mathrm{II}}=\left\{\left(0^{(k)}, 0^{(k)}\right) \mid F^{P}\left(0^{(k)}\right)=\max _{k} F\left(0^{(k)}\right)\right\} .
$$

The rule can be interpreted simply as follows. For occupation of an empty cell, those who can equally reduce their potentials to the fullest extent by moving to this cell share the same probability. For occupation of a cell that has been occupied by one pedestrian, those with the same maximal projection of the aggregated force on the moving direction share the same probability.

\section{Simulation}

In the simulation, the density is scaled to its maximum $\rho_{\max }^{*}=12.5 \mathrm{ped} / \mathrm{m}^{2}$, and length is scaled to $0.4 \mathrm{~m}$. The moving speed is set as $v_{\max }=1 \mathrm{~m} / \mathrm{s}$, and thus each time step for update is approximately $0.4 \mathrm{~s}$. The scaled radius $R$ is taken to be 3.5 along the level or vertical direction and $3.5 \sqrt{2}$ along the diagonal directions which, excluding the center cell, suggests three layer cells in the visible range. The cost distribution $c_{a}(x, y, t)$ is given by

$$
c_{a}(x, y, t)=1+g_{0} \rho^{\gamma}(x, y, t),
$$

where $g_{0}=0.075$ and $\gamma=2$.

The formulations for the perceived cost potential field with and without the aggregated force field are called Models 1 and 2, respectively, and are implemented for comparison. We note that Model 2 is a simple extension of the formulation in Zhang et al. (2012) that takes visibility into account.It also differs from Model 1 in that a cell may not be occupied 
by two pedestrians.

\subsection{Pedestrian flow through a bottleneck}

Pedestrians enter a corridor from left to right with an inflow rate of $P_{0} \in[0,1]$. More precisely, each cell adjacent to the entrance is occupied by one pedestrian with the probability $P_{0}$ in each time step, provided that the cell is empty. The corridor is divided into left and right areas, which can be further divided into $10 \times 12$ and $10 \times 6$ cells, respectively. Initially, the corridor is empty. We are concerned with the pedestrian flow near the central vertical line or bottleneck.

The simulation proceeds for 100 time steps for a range of $P_{0}$ from 0.1 to 1 , and the average flow at the front of the bottleneck over the interval between time steps 30 and 90 is computed. We note here that the pedestrian flow had arrived at the bottleneck before $t=30$ and that the flow is defined as the number of pedestrians crossing a fixed location of the walking domain per unit time and unit distance. Fig. 4 shows the relationship between the inflow rate $P_{0}$ and the average flow at the entrance of the bottleneck. In Fig. 4(a), we observe little difference between Models 1 and 2 for the application of open boundary conditions at the exit (i.e. pedestrians are removed after arrival). In this case, the average flow at the bottleneck is increasing with increasing inflow rate $P_{0}$ until $P_{0} \approx 0.3$; it reaches the capacity of nearly $3 \mathrm{ped} / \mathrm{m} / \mathrm{s}$ for $P_{0} \approx 0.3$, at which point the bottleneck takes effect on the pedestrian flow. That Models 1 and 2 produce similar results suggests that most of the cells are not over-crowded (or occupied by two pedestrians) for the simulation by Model 1 .

(a)

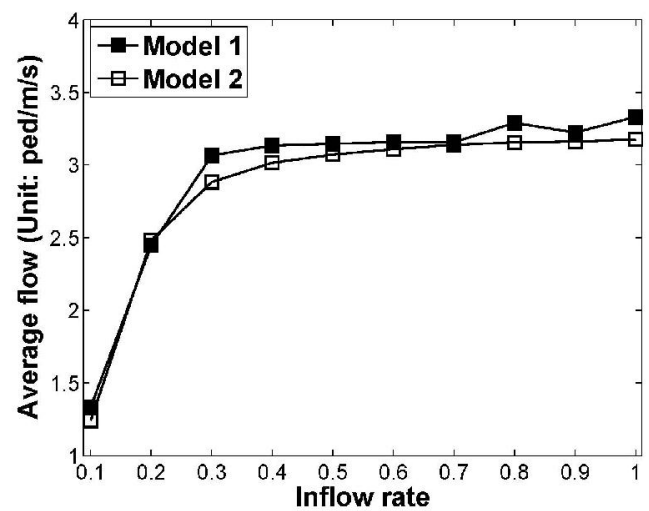

(b)

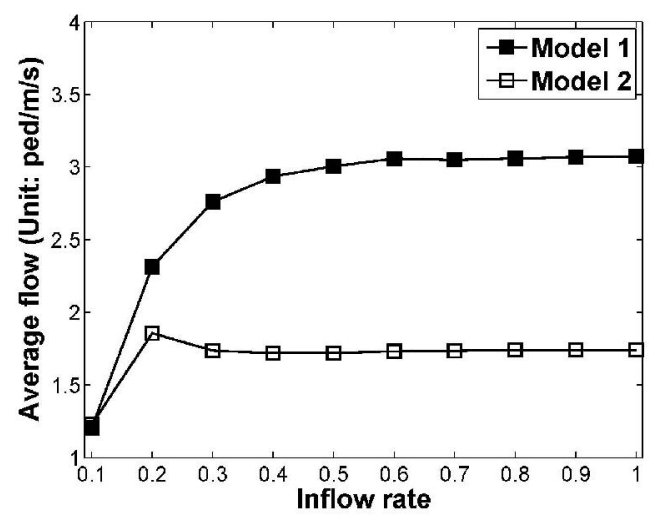

Fig. 4. Plot of the average flow at the entrance of the bottleneck against the inflow rate: (a) the exit is open all the time; (b) the exit is open after 49 time steps. 
To simulate crowded or over-crowded pedestrian flow, solid-wall boundary conditions are applied at the exit before time step $t=50$ and the exit is open afterward. For $P_{0} \geq 0.2$, Model 1 produces a much higher average flow at the bottleneck than Model 2, as shown in Fig. 4(b). We note that both Models 1 and 2 similarly suggest three phases as follows: (1) pedestrian flow arrives and then reaches capacity at the bottleneck; (2) jams moving backward from the exit begin to cross the bottleneck with zero flow thereafter; and (3) dissipation (which is similar to a rarefaction wave) moving backward after $t=50$ from the exit begins to cross the bottleneck and the capacity recovers at the bottleneck. In the first and third phases, Models 1 and 2 produce almost the same capacity, which is approximately equal to that in Fig. 4(a). In the second phase, the interval in which the back-moving jam arrives at the bottleneck, which is simulated by Model 1, is twice that simulated by Model 2, asthe jam density in Model 1 is twice that in Model 2. This suggests a much longer interval for the second phase and thus a much larger average flow at the bottleneck for the simulation of Model 1 than for Model 2. Fig. 5 shows the evolution of three phases for comparison between Models 1 and 2.

(a)

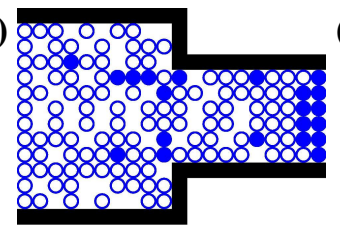

(e)

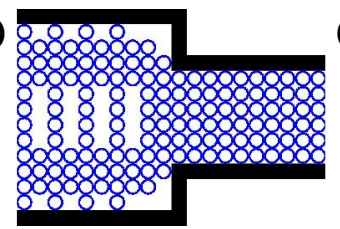

(b)

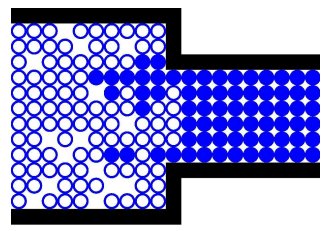

(f)

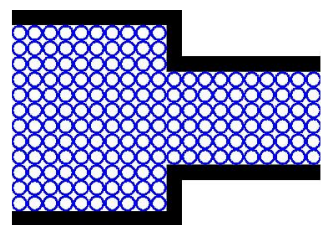

(c)

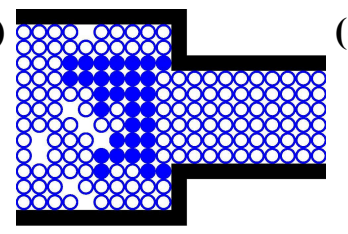

(g)

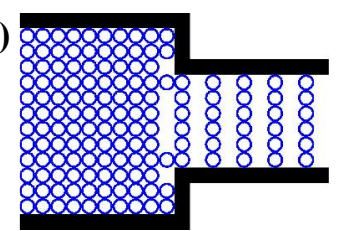

(d)

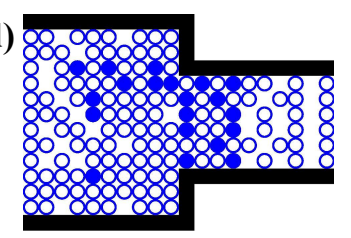

(h)

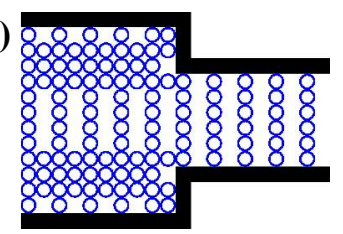

Fig. 5. Evacuation process simulated by the perceived potential field model with the aggregated force field, i.e. Model 1 (a-d); and without the aggregated force field, i.e. Model 2 (e-h), at time steps 30, 49, 60 and 90 within 100 time steps. The exit is opened after 49 time steps and the inflow rate is $P_{0}=1$.

The fundamental diagrams are plotted in Fig. 6 through simulation by Models 1 and 2 . By using both the open boundary condition and the periodic boundary condition, each of the two simulations is implemented for 1000 time steps. For the open condition, the dimensionless densities are set initially in the walking domainto be $\rho_{0}=1$ for Model 1 and $\rho_{0}=0.5$ for Model 2, and the inflow rate is set to be 


$$
P_{0}= \begin{cases}1, & \text { time step } \in[0,200), \\ 0.5, & \text { time step } \in[200,400), \\ 0.2, & \text { time step } \in[400,600), \\ 0.1, & \text { time step } \in[600,1000]\end{cases}
$$

For the periodic boundary condition, the density is initially set in a range from 0.1 to 0.9 , each of which corresponds to a simulation. Density and flow data for drawing the fundamental diagram are taken as averages over five consecutive time steps and $4 \times 4$ cells constituting a middle area in the narrowed corridor. The density is defined as the ratio of the number of pedestrians in the middle area to the number 32 representing occupation to the fullest extent; the flow is defined as the division of the number of pedestrians crossing the mid-perpendicular segment in a time step by the number 4 representing the involved cells.

(a)

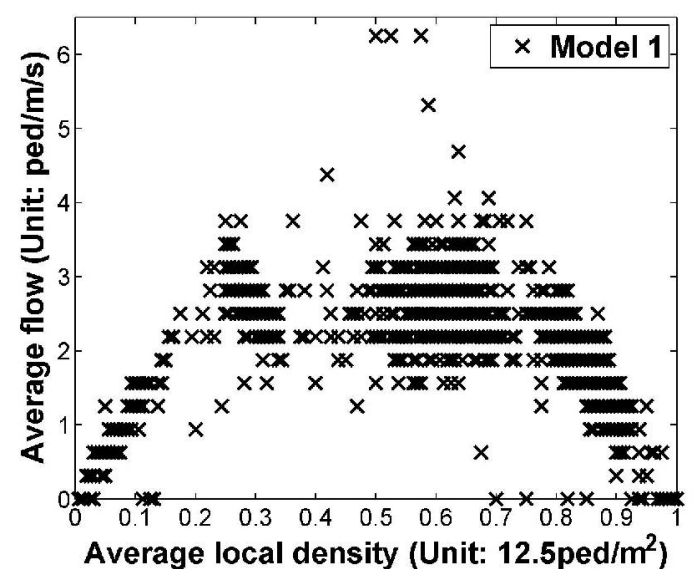

(b)

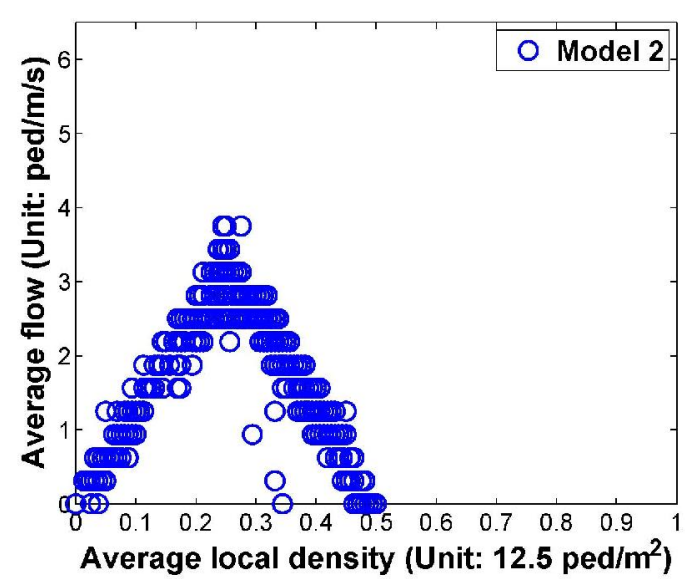

Fig. 6. Fundamental diagrams of (a) Model 1 and (b) Model 2 for $v_{\max }=1 \mathrm{~m} / \mathrm{s}$, measured in a middle section consisting of $4 \times 4$ cells in the narrowed corridor.

Fig. 6(a) is plotted through simulation by Model 1; it shows two flow peaks for crowed and over-crowded pedestrian flows and is similar to that of Helbing et al. (2007). The first flow peak reflects the phase transition from free flow to crowded flow. In this case, the density approaches $6.25 \mathrm{ped} / \mathrm{m}^{2}$ and there is no freespace for pedestrians to move as in a normal situation. The pushing forces are aggregated to a critical value and each pedestrian has to move to a neighboring cell that has already been occupied by another pedestrian; thus the second flow peak appears. The fundamental diagram provides a reasonable demonstration that pedestrians in over-crowded situation are able to keep moving successively. In contrast, the fundamental diagram plotted through simulation by Model 2, as shown in Fig. 
6(b), does not reflect a realistic flow-density relationship, and is similar to that simulated by the floor field model (Kirchner et al., 2004, Schadschneider and Seyfried, 2009) for the restriction that a cell can be occupied by at most one pedestrian.

\subsection{A corridor with a $90^{\circ}$ corner}

The corridor is a connection of two passageways, each of which includes $6 \times 10$ cells. The corridor is divided into four areas, labeled A, B, C, and D, and the entrance and exit are located in the bottomleft and topright ends of the corridor, respectively (Fig. 7). Initially, pedestrians are randomly distributed in the corridor with the density $\rho_{0}$, and the open boundary condition is applied during the simulation.

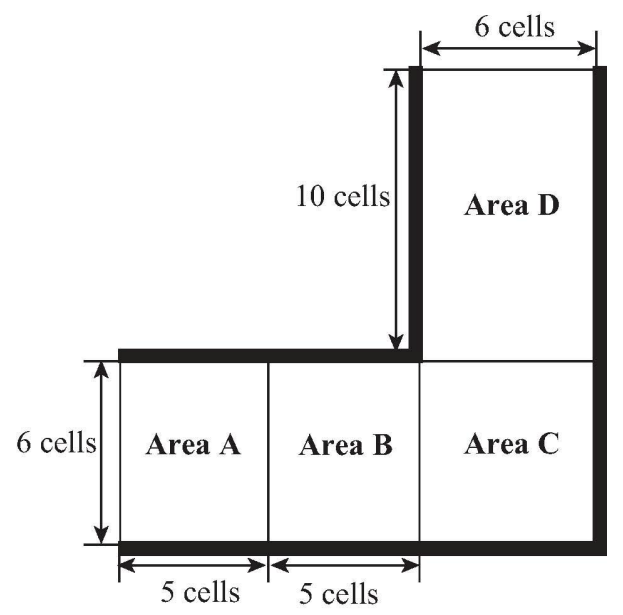

Fig. 7. Sketch of the corridor with a $90^{\circ}$ corner and its local areas.

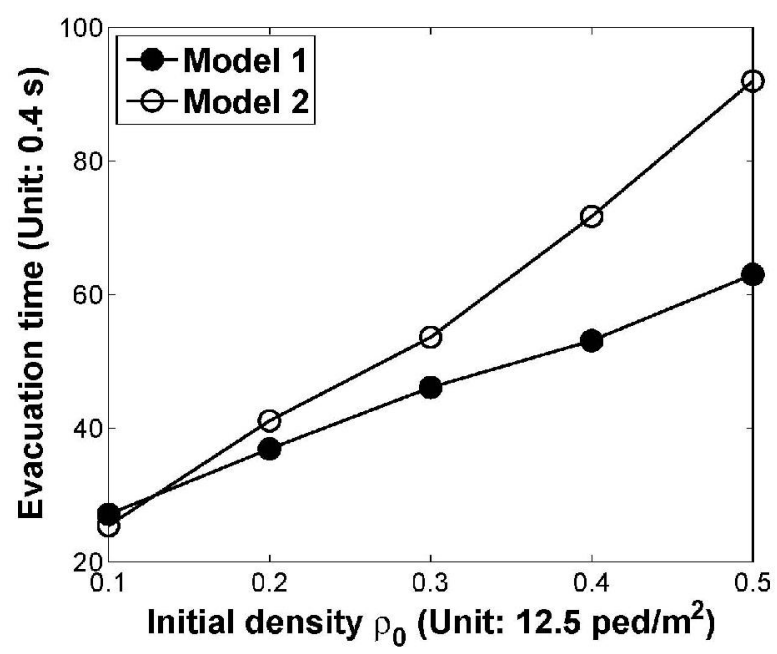

Fig. 8. Relation of pedestrian evacuation time to initial pedestrian density.

The evacuation time is recorded for comparison between Models 1 and 2. Fig. 8 shows that the evacuation time increases with increasing initial density $\rho_{0}$ for the simulation by Models 1 and 2. Moreover, the evacuation simulated by Model 1 is more efficient than that simulated by Model 2, which indicates that the aggregated force field will probably reduce the travel time. The results produced by Model 1 are in accordance with common sense.

For the initial density $\rho_{0}=0.5$, which implies that each cell is occupied by one pedestrian, Fig. 9 shows the contours of pedestrian flow in time steps 20, 30, 40 and 50 . These indicate that both Models 1 and 2 are able to simulate the "corner hugging" phenomenon, which is similar to that in Still (2000), Steffen and Seyfried (2009) and Dias 
et al. (2012). Moreover, both Models 1 and 2 reflect reasonably well the collection effects in uncrowded pedestrian flow. We note that the extremely high density at the turning of the corner agrees well with observation, which is simulated by Model 1 (Figs. 9(a)-9(d)). However, such an over-crowding situation cannot be reproduced by Model 2; see Figs. 9(e)-9(h) for comparison.

(a)

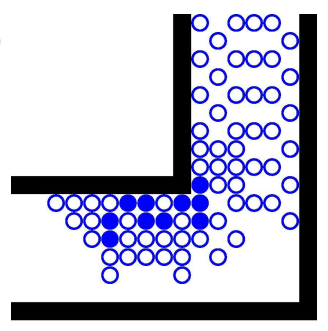

(e)

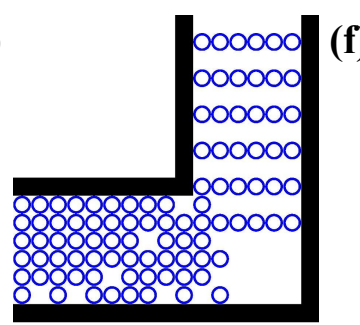

(b)

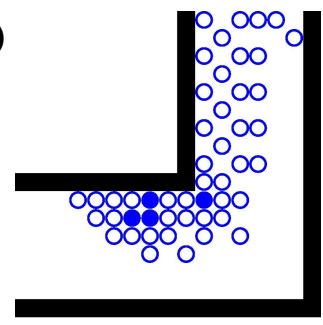

(f)

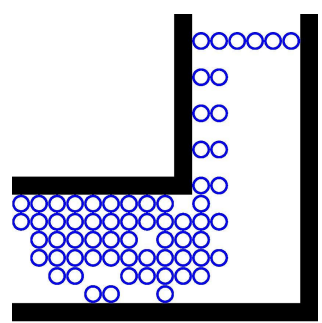

(c)

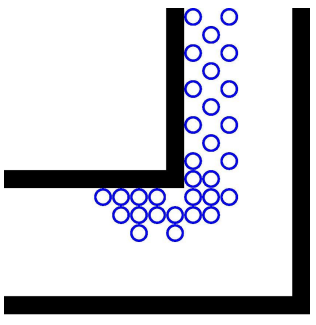

(g)

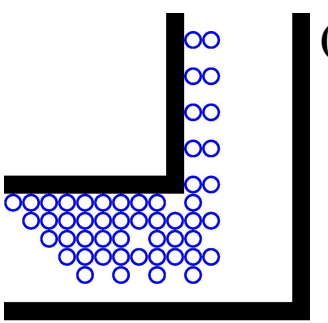

(d)

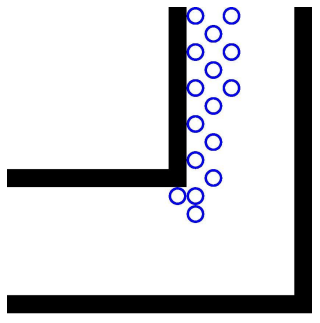

(h)

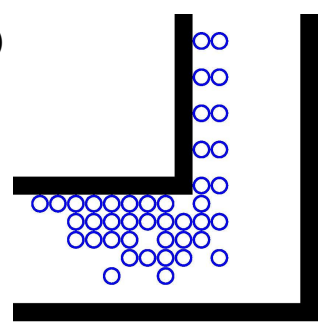

Fig. 9. Evacuation process simulated by the perceived potential field model with the aggregated force field, i.e. Model 1 (a-d); and without the aggregated force field, i.e. Model 2 (e-h), at time steps 20, 30, 40 and 50. Here, $\rho_{0}=0.5$.

We are further interested in the maximal densities in those areas shown in Fig. 7 for the following simulation that proceeds for 100 time steps. Initially, all cells are assumed to be empty and pedestrians begin to enter the domain with inflow rate $P_{0}=1$. The maximal densities in Areas A, B, C and D are recorded in each time step and are shown in Fig. 10. For Model 1, we observe that the appearance of an over-crowded situation is quite frequent in Area B (Fig. 10(b)); as is also the case with Area A (Fig. 10(a)) due to the propagation of over-crowded flow from Area B. There are no over-crowded cells appearing in Areas C and D, as shown in Figs. 10(b) and 10(a), respectively. The turning of the corner (Area B) actually functions somewhat as a bottleneck due to poor visibility and the resulting pushing forces in that area. The conclusion is significant for the study of some special areas which are prone to being over-crowded and of stampedes; see also Dias et al. (2012) for reference. Conversely, no over-crowded areas are observed throughout the simulation by Model 2 (see Figs. 10(a) and 10(b)). 
(a)

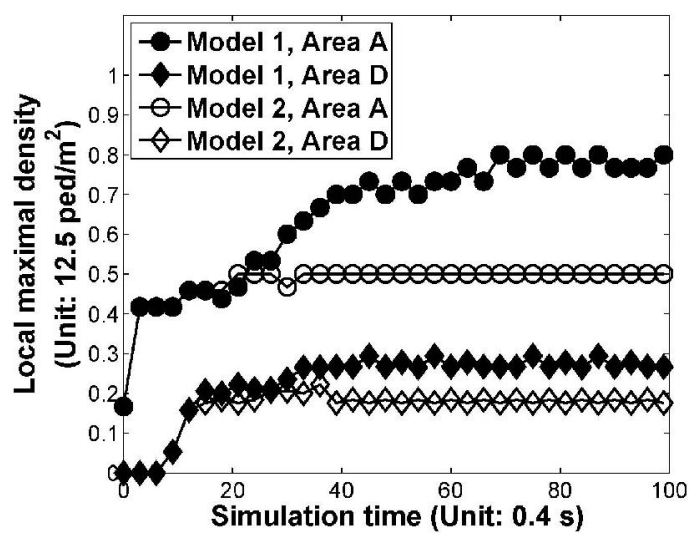

(b)

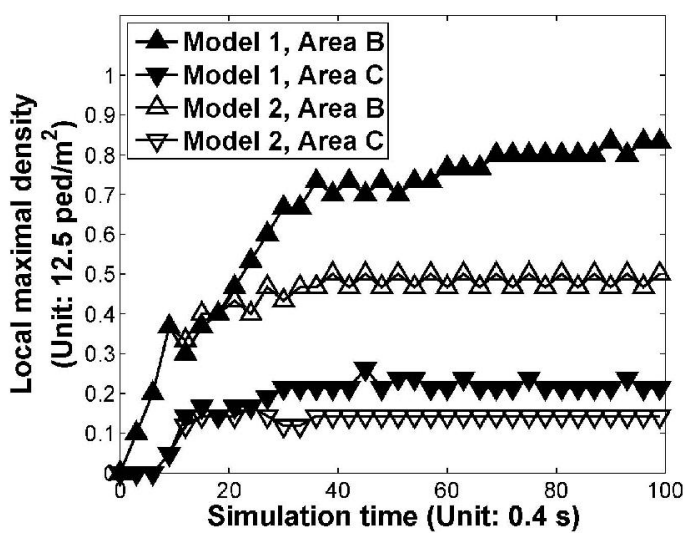

Fig. 10. Plot of local maximal density against time steps (a) in Areas A and D, and (b) in Areas B and C, according to the four local areas shown in Fig. 7. Here, the inflow rate is $P_{0}=1$ and the total time steps are 100 .

\section{Conclusion}

We extend the cost potential field model in Zhang et al. (2012) to describe pedestrian flow in a domain with poor visibility or complex geometries, by proposing a perceived cost potential field and an aggregated force field. Allowing a cell to be occupied by, at most, two pedestrians, the proposed model can simulate over-crowded pedestrian flow with a maximal density of $12.5 \mathrm{ped} / \mathrm{m}^{2}$. This demonstrates an advantage of the proposed model over many classical CA models that allow the occupation of a cell by only one pedestrian or a much lower maximal density of $6.25 \mathrm{ped} / \mathrm{m}^{2}$.

Moreover, the proposed aggregated force field is indicated to be critical for the simulation of over-crowded flows in some special areas, such as those near a bottleneck or at the turning of a corner. As was investigated in Helbing et al. (2007), special areas are vulnerable to being over-crowded due to crowded flow and typically result in disaster. Such special areas are associated with complex geometries or poor visibility in our formulation, and the first phase of development has been reflected well in the simulation. Therefore, the proposed model can be used for the design of a walking facility or to predict danger requiring management.

\section{Acknowledgements}

The work described in this paper was jointly supported by the National Natural Science 
Foundation of China (Grant No. 11072141, 11272199, 71125004), the National Basic Research Program of China (2012CB725404), the Shanghai Program for Innovative Research Team in Universities, the Research Grants Council of the Hong Kong Special Administrative Region, China (Grant Nos. HKU7184/10E and HKU7175/12E), and a National Research Foundation of Korea grant funded by the Korean government (MEST) (NRF-2010-0029446).

\section{References}

Alizadeh, R., 2011. A dynamic cellular automaton model for evacuation process with obstacles. Safety Science 49, 315-323.

Bönisch, C., Kretz, T., 2009. Simulation of pedestrians crossing a street. eprint arXiv, 0911.2902

Burstedde, C., Klauck, K., Schadschneider, A., Zittartz, J., 2001.Simulation of pedestrian dynamics using a two-dimensional cellular automaton. Physica A 295, 507-525.

Dias, C., Sarvi, M., Shiwakoti, N., Burd, M., 2012. Turning angle effect on emergency egress. Transportation Research Record: Journal of the Transportation Research Board $2312,120-127$.

Fruin, J.J., 1993. The causes and prevention of crowd disasters, in: Smith, R.A., Dickie, J.F. (Eds.), Engineering for Crowd Safety, Elsevier, Amsterdam.

Guo, R.Y., Huang, H.J., Wong, S.C., 2012. Route choice in pedestrian evacuation under conditions of good and zero visibility: Experimental and simulation results. Transportation Research Part B 46, 669-686.

Guo, R.Y., Huang, H.J., 2012. Formulation of pedestrian movement in microscopic models with continuous space representation. Transportation Research Part C 24, 50-61.

Guo, R.Y., Tang, T.Q.,2012.A simulation model for pedestrian flow through walkways with corners.Simulation Modelling Practice and Theory21,103-113.

Helbing, D., Buzna, L., Johansson, A., Werner, T., 2005. Self-organized pedestrian crowd dynamics: Experiments, simulations, and design solutions. Transportation Science 39, $1-24$. 
Helbing, D., Johansson, A., Al-Abideen, H.Z., 2007. Dynamics of crowd disasters: An empirical study. Physical Review E 75, 046109.

Helbing, D., Molnár, P., 1995. Social force model for pedestrian dynamics. Physical Review E 51, 4282-4286.

Henein, C.M., White, T., 2007. Macroscopic effects of microscopic forces between agents in crowd models. Physica A 373, 694-712.

Henein, C.M., White, T., 2010. Microscopic information processing and communication in crowd dynamics. Physica A 389, 4636-4653.

Hoogendoorn, S.P., Bovy, P.H.L., 2004. Pedestrian route-choice and activity scheduling theory and models. Transportation Research Part B 38, 169-190.

Huang, H.J., Guo, R.Y., 2008. Static floor field and exit choice for pedestrian evacuation in rooms with internal obstacles and multiple exits. Physical Review E 78, 021131.

Huang, L., Wong, S.C., Zhang, M.P., Shu, C.W., Lam, W.H.K., 2009. Revisiting Hughes' dynamic continuum model for pedestrian flow and the development of an efficient solution algorithm. Transportation Research Part B 43, 127-141.

Hughes, R.L., 2002. A continuum theory for the flow of pedestrians. Transportation Research Part B: Methodological 36, 507-535.

Jiang, Y.Q., Zhang, P., Wong, S.C., Liu, R.X., 2010. A higher-order macroscopic model for pedestrian flows. Physica A 389, 4623-4635.

Kirchner, A., Klüpfel, H., Nishinari, K., Schadschneider, A., Schreckenberg, M., 2004. Discretization effects and the influence of walking speed in cellular automata models for pedestrian dynamics. Journal of Statistical Mechanics: Theory and Experiment 2004, P10011.

Kirchner, A., Schadschneider, A., 2002. Simulation of evacuation processes using a bionics-inspired cellular automaton model for pedestrian dynamics. Physica A 312, 260-276. 
Kretz, T., Bönisch, C., Vortisch, P., 2010. Comparison of various methods for the calculation of the distance potential field, in: Klingsch, W.W.F., Rogsch, C., Schadschneider, A., Schreckenberg, M. (Eds.), Pedestrian and Evacuation Dynamics 2008, Springer-Verlag, Berlin Heidelberg. pp. 335-346.

Kretz, T., 2009. The use of dynamic distance potential fields for pedestrian flow around corners. eprint arXiv, 0906.2667.

Kretz, T., 2010. The dynamic distance potential field in a situation with asymmetric bottleneck capacities, in: Bandini, S., Manzoni, S., Umeo, H., Vizzari, G. (Eds.), Cellular Automata. Springer-Verlag, Berlin Heidelberg. Volume 6350 of Lecture Notes in Computer Science, pp. 480-488.

Kuang, H., Li, X.L., Song, T., Dai, S.Q., 2008. Analysis of pedestrian dynamics in counter flow via an extended lattice gas model. Physical Review E 78, 066117.

Lee, R.S.C., Hughes, R.L., 2005. Exploring trampling and crushing in a crowd. Journal of Transportation Engineering 131, 575-582.

Moussaïd, M., Helbing, D., Theraulaz, G., 2011. How simple rules determine pedestrian behavior and crowd disasters. Proceedings of the National Academy of Sciences 108, 6884-6888.

Peng, Y.C., Chou, C.I., 2011. Simulation of pedestrian flow through a "T" intersection: A multi-floor field cellular automata approach. Computer Physics Communications 182, 205-208.

Schadschneider, A., Seyfried, A., 2009. Validation of CA models of pedestrian dynamics with fundamental diagrams. Cybernetics and Systems 40, 367-389.

Schadschneider, A., 2002. Cellular automaton approach to pedestrian dynamics - theory, in: Schreckenberg, M., Sharma, S. (Eds.), Pedestrian and Evacuation Dynamics, Springer, Berlin Heidelberg. pp. 75-86.

Steffen, B., Seyfried, A., 2009. Modelling of pedestrian movement around 90 and 180 degree bends. eprint arXiv, 0912.0610.

Still, G.K., 2000. Crowd Dynamics. Ph.D. thesis. University of Warwick. 
Varas, A., Cornejo, M.D., Mainemer, D., Toledo, B., Rogan, J., Muñoz, V., Valdivia, J.A.,2007. Cellular automaton model for evacuation process with obstacles. Physica A $382,631-642$.

Xia, Y.H., Wong, S.C., Shu, C.W., 2009. Dynamic continuum pedestrian flow model with memory effect. Physical Review E 79, 066113.

Xie, D.F., Gao, Z.Y., Zhao, X.M., Wang, D.Z.W., 2012. Agitated behavior and elastic characteristics of pedestrians in an alternative floor field model for pedestrian dynamics. Physica A 391, 2390-2400.

Xiong, T., Zhang, P., Wong, S.C., Shu, C.W., Zhang, M.P., 2011. A macroscopic approach to the lane formation phenomenon in pedestrian counterflow. Chinese Physics Letters 28, 108901.

Xu, Y., Huang, H.J., 2012. Simulation of exit choosing in pedestrian evacuation with consideration of the direction visual field. Physica A 391, 991-1000.

Zeng, J.B., Leng, B., Xiong, Z., Qin, Z., 2011. Pedestrian dynamics in a two-dimensional complex scenario using a local view floor field model. International Journal of Modern Physics C 22, 775-803.

Zhang, P., Jian, X.X., Wong, S.C., Choi, K., 2012. Potential field cellular automata model for pedestrian flow. Physical Review E 85, 021119.

Zhao, H.K., 2005. A fast sweeping method for eikonal equations. Mathematics of Computation 74, 603-627. 